Espacio, Tiempo y Forma, Serie II, Historia Antigua, t. 25, 2012, pp. 75-92

\title{
Una donación ex testamento procedente del foro de Los Bañales (Uncastillo, Zaragoza)
}

\author{
A donation ex testamento from the forum of Los Bañales \\ (Uncastillo, Zaragoza)
}

\author{
ÁNGEL A. JORDÁN LORENZO*
}

RESUMEN

El objetivo de este artículo es presentar un conjunto de pedestales cultuales encontrados in situ en el foro de la ciudad romana de Los Bañales (Uncastillo, Zaragoza), y financiados ex testamento por Pom(peia ?) Pullatis $f$. Paulla.

\section{PALABRAS CLAVE}

Epigrafía, Inscripciones Latinas, Los Bañales (Uncastillo, Zaragoza), luppiter, Nymphae, Matres, Apolo.

\section{ABSTRACT}

The aim of this paper is to present a set of cultual bases found in situ in the forum of the Roman city of Los Bañales (Uncastillo, Zaragoza), and financed ex testamento by Pom(peia ?) Pullatis $f$. Paulla.

\section{KEYWORDS}

Epigraphy, Latin Inscriptions, Los Bañales (Uncastillo, Zaragoza), luppiter, Nymphae, Matres, Apolo.

Recibido el 13 de agosto de 2012. Aceptado el 11 de septiembre de 2012

Los Bañales de Uncastillo (Zaragoza) era una ciudad romana emplazada en la actual comarca de las Cinco Villas, al pie de una calzada estratégica que permitía la unión de Caesaraugusta y, por ende, el valle del Ebro, con el Sur de la Galia, en su bifurcación hacia el Este tras llegar a la ciuitas emplazada en Campo Real/Fillera (Sos del Rey Católico/Sangüesa) ${ }^{1}$, y, por otro lado, con el

* Archivo Epigráfico de Hispania. E-mail: ajorlor@yahoo.es. Quisiera expresar mi agradecimiento a los Dres. Javier Andreu Pintado, Joaquín Gómez-Pantoja, Francisco Beltrán Lloris, y a José-Vidal Madruga por la ayuda y sugerencias dadas a la hora de leer e interpretar estas inscripciones.

1 En general sobre esta vía uid. MORENO GALLO, Isaac, Item a Caesarea Augusta Beneharno. La carretera romana de Zaragoza al Bearn, Ejea de los Caballeros, Institución Fernando el Católico, 2009. También resultan interesantes los trabajos anteriores de AGUAROD, Carmen y LOSTAL, Javier, "La vía romana de las Cinco Villas», Caesaraugusta, 55-56, (1982), pp. 167-218 y MAGALLÓN BOTAYA, María Ángeles «La red viaria romana en las Cinco Villas», en: Actas de las I Jornadas de Estudio sobre las Cinco Villas (Ejea, diciembre 1985), Zaragoza, Centro de Estudios de las Cinco Villas, 1986, pp. 95143, si bien el tramo Los Bañales - Cara, en nuestra opinión, resulta excesivamente sobrevalorado pues debió de tratarse de una vía secundaria, cf. MORENO GALLO, Isaac, Item a Caesarea..., p. 117. 
Cantábrico, pasando por Pompelo, en su desvío hacia el Oeste desde Campo Real/Fillera ${ }^{2}$.

La ciudad se extiende a lo largo de $24 \mathrm{Ha}^{3}$ en la parte central de un valle comprendido entre los cerros de EI Pueyo, al Oeste, Puy Foradado, al Este, y El Huso y La Rueca, al Sur, mientras que al Norte limita con el barranco de Valdebañales. En este espacio y, más en concreto, en la ladera Este de El Pueyo, la Fundación Uncastillo, por encargo del Gobierno de Aragón, viene realizando desde hace cuatro años excavaciones arqueológicas en dos zonas correspondientes a un barrio doméstico-artesanal, situado en las inmediaciones de un pequeño recinto ter$\mathrm{mal}^{4}$, y en el foro de la ciudad ${ }^{5}$. Los trabajos en este último espacio se han centrado en su pórtico Oeste descubriéndose, a lo largo de las campañas de 2011 y 2012, la existencia de, al menos, tres sacella, dos con forma de ediculae y uno con la de una exedra, que se abren hacia el Oeste. Dos de ellos, la exedra y el edículo derecho, han permitido recuperar un impresionante conjunto de hasta nueve inscripciones in situ, cuatro en la exedra y cinco en el edículo, en la actualidad en proceso de estudio, centrándose la atención en las siguientes páginas, en el primero de ellos, compuesto por cuatro pedestales.

\section{EL RECINTO DE LA EXEDRA}

Con el nombre de «exedra» se identifica un espacio cuadrangular cerrado por un muro semicircular situado al final del pórtico occidental, marcando una transición entre el cierre cubierto por el doble pórtico y una zona abierta, fruto de una ampliación hacia el Sur realizada en fecha, por el momento, desconocida (figs. 1 y 2). Este es-

2 Sobre la ciuitas emplazada en Campo Real/Fillera (Sos del Rey Católico/Sangüesa), quizá identificable con la antigua Arsi, uid. ANDREU PINTADO, Javier, ARMENDÁRIZ MARTIJA, Javier, OZCÁRIZ GIL, Pablo, GARCÍA-BARBERENA UNZU, María y JORDÁN LORENZO, Ángel A., «Una ciudad de los vascones en el yacimiento de Campo Real/Fillera (Sos del Rey Católico-Sangüesa)», AEspA, 81, (2008), pp. 75100; ANDREU PINTADO, Javier, JORDÁN LORENZO, Ángel A. y ARMENDÁRIZ MARTIJA, Javier, «Nuevas aportaciones a la epigrafía de Campo Real/Fillera (Sos del Rey Católico-Sangüesa)», Zephyrus, 65, (2010), pp. 179-198; ANDREU PINTADO, Javier, ZUAZÚA, Nicolás, ARMENDÁRIZ MARTIJA, Javier y ROYO PLUMED, Hernando, «A propósito de una cornucopia romana en mármol procedente del territorio de la ciudad romana de Campo Real / Fillera (Sangüesa, Navarra)», VII Congreso General de Historia de Navarra, vol. 1., Príncipe de Viana, 253, (2011), pp. 97-120 ó ANDREU PINTADO, Javier, LASUÉN ALEGRE, María, MAÑAS ROMERO, Irene y JORDÁN LORENZO, Ángel A., «Novedades de arte romano provincial en territorio vascón: un mosaico marino inédito procedente de Campo Real/Fillera (Sos del Rey Católico/Sangüesa)», en: NOGALES, Trinidad y RODÀ, Isabel (eds.), Roma y las provincias: modelo y difusión. XI Coloquio internacional de arte romano provincial, Roma, L'Erma di Bretschneider, 2011, pp. 839-850.

3 Sobre los límites de la ciudad uid. ANDREU PINTADO, Javier, GONZÁLEZ SOUTELO, Silvia, GARCÍA ENTERO, Virginia, JORDÁN LORENZO, Ángel A. y LASUÉN ALEGRE, María, «Cuestiones urbanísticas en torno a la ciuitas de los Bañales (Uncastillo, Zaragoza)», SPAL, 17, (2008), pp. 233-266.

4 Vid. URIBE AGUDO, Paula, HERNÁNDEZ VERA, Jose Antonio y BIENES CALVO, Juan José, «La edilicia urbana privada en Los Bañales: estado de la cuestión», en: ANDREU PINTADO, Javier (ed.), La ciudad romana de Los Bañales (Uncastillo, Zaragoza): entre la historia, la arqueología y la historiografía, Caesaraugusta, 82 (2011), pp. 241-260, esp. 253-260; ANDREU PINTADO, Javier, La ciudad romana de Los Bañales. Uncastillo - Zaragoza, Zaragoza, Prames, 2012, pp. 56-61.

5 Un pequeño avance de las excavaciones realizadas en el foro puede verse en ANDREU PINTADO, Javier, La ciudad romana..., pp. 44-48. 
pacio ha sido muy castigado por los expoliadores, ya desde época antigua, quedando en la mayor parte del recinto apenas una o dos hiladas de los sillares de sus muros, con la excepción del de cierre por el Oeste, que conserva tres hiladas. Este saqueo ha dejado diversas marcas en la forma de varios sillares fracturados al inicio de cada uno de los lienzos conservados (rotura que, por otra parte, impidió que siguieran retirándose los muros favoreciendo su conservación), así como un nivel de gravas y cantos rodados que, posiblemente, tuvo el objetivo de cerrar y nivelar la zanja que se realizó para poder extraer los sillares del muro de cierre Oeste.

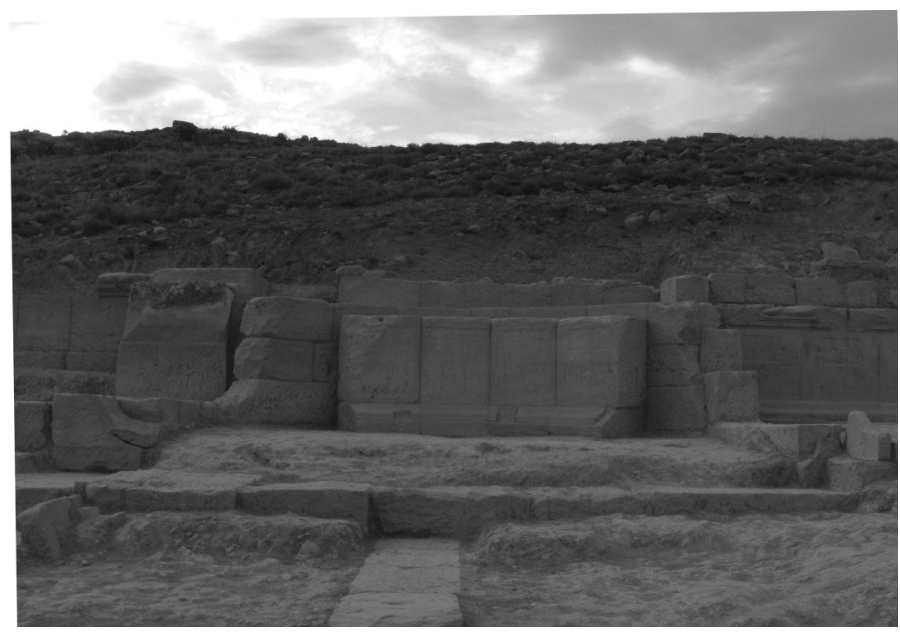

Fig. 1. La exedra del pórtico occidental

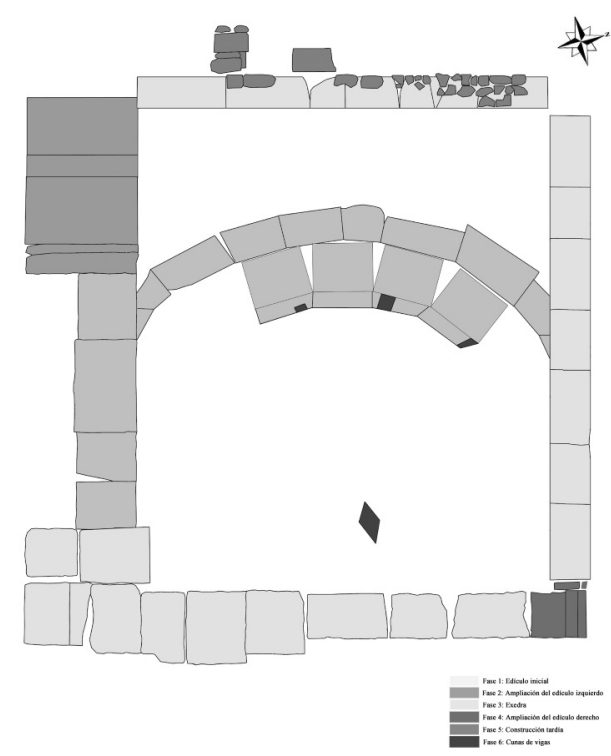

Fig. 2. Plano de la exedra 
En general, el espacio de la exedra presenta un área interior de $16 \mathrm{~m}^{2}(4,08 \mathrm{~m}$. $x 4,8 \mathrm{~m}$.) y se construyó amortizando un espacio cuadrangular previo, de características desconocidas, pero con unas dimensiones interiores de, al menos, 5,02 m. x 4,8 m. (fig. 2 y 5).

De este espacio primitivo se ha podido identificar el muro de cierre trasero, realizado con sillares de $40 \mathrm{cms}$. de grosor y diversas anchuras. Este muro, posiblemente, se asentó directamente sobre la roca madre, que en esta zona coincide con un sustrato geológico de carácter arcilloso, lo cual ha originado su compactación por el peso recibido, como se aprecia en el ligero hundimiento de la parte central de este muro.

También es posible atribuir a esta primera fase constructiva el muro de cierre derecho, pues, aunque se encuentra perdido hasta las primeras hiladas, la factura de los sillares conservados es similar a la de los anteriores. Sin embargo, éste no acaba de cerrar con el posterior, faltando un sillar, posiblemente como consecuencia del expolio (fig. 3). Esta situación invita a considerar que este recinto correspondiente a una primera fase, quizá tuvo una continuación hacia el Norte, siendo amortizado por la posterior construcción del edículo derecho, si bien hasta que no se termine de excavar este aspecto no podrá aclararse. Además, en uno de los sillares se conservan restos de argamasa destinada al enlucido de, como mínimo, este muro. En relación con ello, no se puede descartar que todas las paredes de la exedra estuvieran enlucidas y pintadas, habiéndose localizado, en un nivel cercano al de circulación, pequeños restos de pintura de color burdeos y azul celeste. Por último, en su parte inferior se ve modificado por la construcción del acceso al edículo derecho. Además, no se descarta que también a este primer momento constructivo pueda atribuirse la hilada de cimentación del cierre del edículo primitivo con el pórtico, como se aprecia en la superposición de la segunda hilada de sillares del muro Sur.

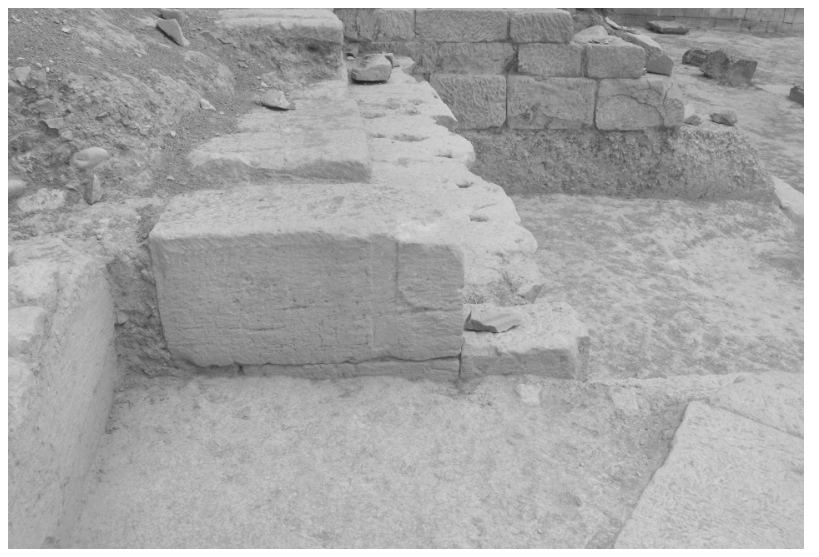

Fig. 3: Detalle de la unión de los muros O(1) y N 


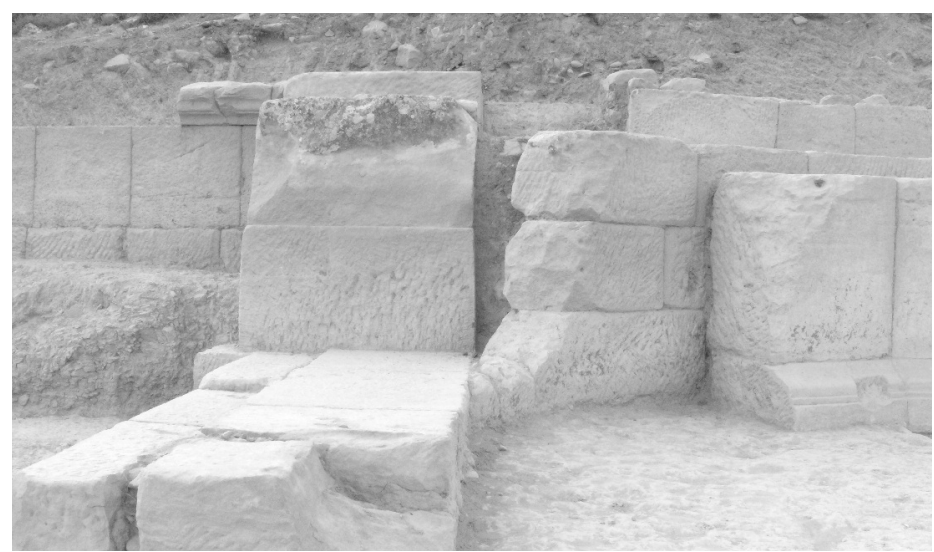

Fig. 4: El muro de cierre por el Sur

El muro Sur de la exedra se construyó amortizando, en parte, un lienzo de grandes sillares de $132 \mathrm{cms}$. de anchura, por un fondo y altura variables, que sirve de cierre a la ampliación del edículo izquierdo (fig. 4), y que es sustituido, a la altura del cierre del muro curvo de la exedra, por un doble muro de sillares. El interior de $112 \times 74$, y el exterior, que cerraría a su vez el edículo izquierdo, de $112 \times 20 \mathrm{cms}$.

Por último, cierra la exedra el muro Oeste, curvo, realizado con sillares de dimensiones desiguales pero, exceptuando el segundo sillar del lado derecho, cortados de forma paralelepípeda, de tal forma que su disposición para formar la curva deja pequeños espacios en su parte posterior, aunque se cuidó que el frente, cara que sería vista, cerrara perfectamente. La construcción de este muro implicaría la amortización del anterior lienzo de cierre, de trazado recto, pues se dispuso a $94 \mathrm{cms}$. por delante de éste (fig. 5).

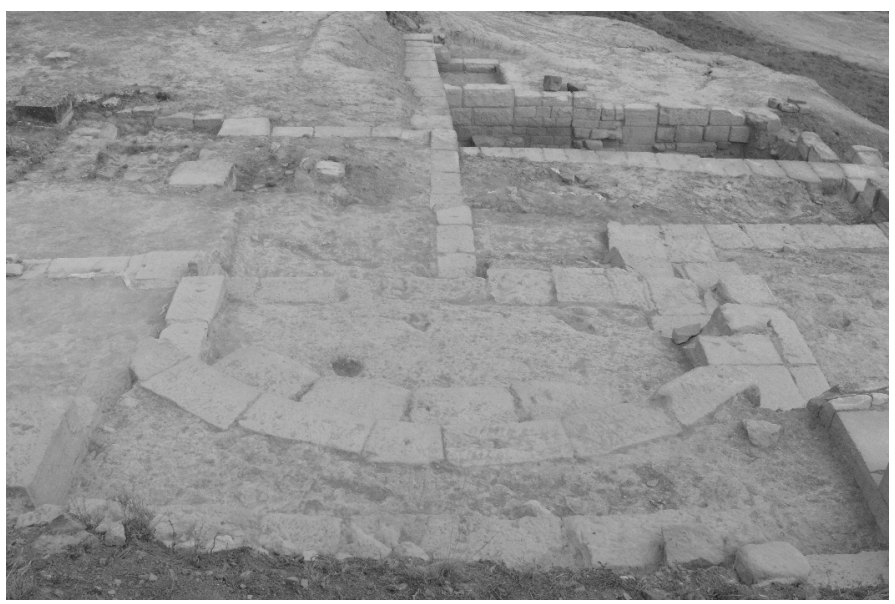

Fig. 5: Vista O-E de la exedra 


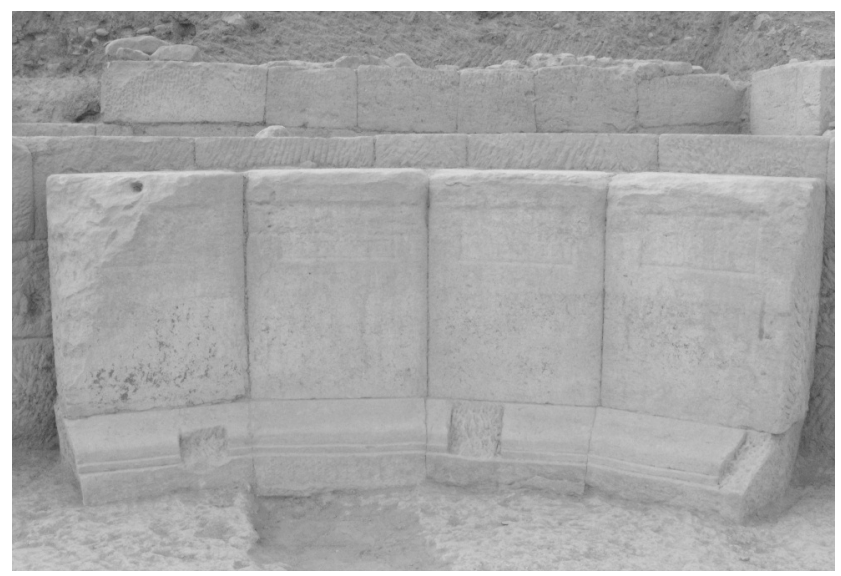

Fig. 6. Detalle del zócalo de los pedestales de la exedra

Apoyados contra este muro de sillares curvos se encuentran cuatro pedestales, dispuestos de forma similar al muro, es decir, juntos por su parte delantera, pero separados en la trasera, para así poder seguir el radio de la exedra. Estos pedestales se han montado sobre unos zócalos moldurados que, dado el carácter independiente de ellos, no siempre mantienen el encuadre con los bloques, de tal forma que los dos zócalos de la derecha se muestran descuadrados hacia la izquierda (fig. 6). Además, los zócalos muestran tres rebajes destinados a soportar tres vigas dispuestas en diagonal hacia el centro de la estancia, que acabarían apoyando en un cuarto agujero de poste localizado a $60 \mathrm{cms}$. del muro de cierre. Estas cunas posiblemente estuvieron destinadas a ser los soportes de las vigas de una pequeña cabaña que se dispuso en un momento tardío, ya abandonada la ciudad, aprovechando los pedestales como muros de cierre (fig. 7).

Para finalizar, también a un momento tardío se puede atribuir la existencia de un muro de sillarejo, realizado empleando el muro de cierre del espacio original de la exedra $\left(\mathrm{O}_{(1)}\right)$, como cimentación de un edificio de características desconocidas, pues tan sólo se ha localizado parte de su muro trasero, pero que se extiende hacia el interior de la ladera de El Pueyo, obteniéndose la impresión de que a esa altura quizá puede empezar una nueva terraza, oculta en la actualidad bajo un potente estrato deposicional (fig. 8). 


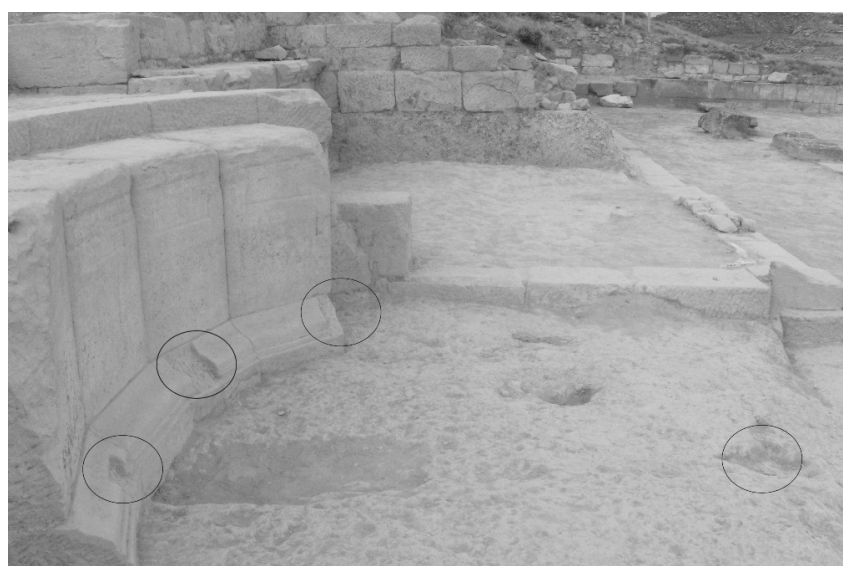

Fig. 7: Último momento de reempleo de la exedra

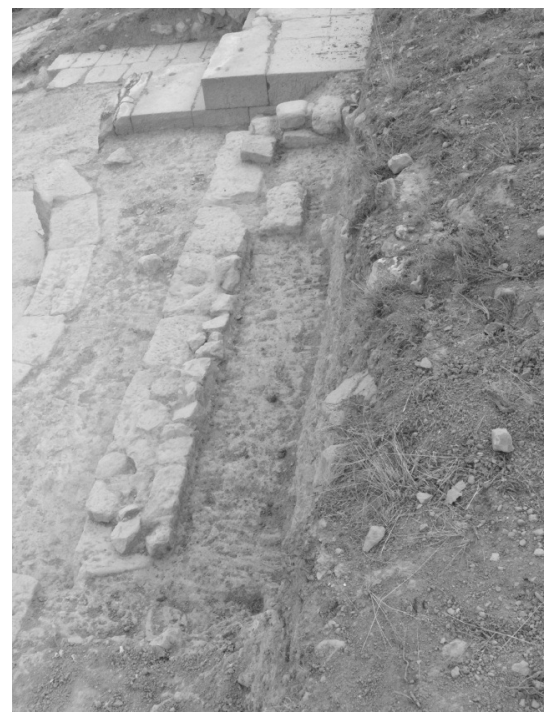

Fig. 8: Construcciones sobre el muro de cierre $O(1)$ de la primera fase

\section{EL CONJUNTO DE PEDESTALES}

Como se ha comentado con anterioridad, en el interior de la exedra se han encontrado cuatro pedestales realizados en arenisca local, que se encuentra muy meteorizada, no pudiéndose descartar que formaran parte de un conjunto mayor en su origen, seis o quizá siete, pues claramente falta uno a la derecha y, al menos, otro a la izquierda. Estos pedestales eran tripartitos, como, posiblemente, fueron también 
los del foro ${ }^{6}$, habiendo perdido todos el coronamiento, como consecuencia del expolio que sufrió la exedra. Las dimensiones totales, iguales en todos los casos, son de 123 x 73 x 58 cms., con un cuerpo central de $95 \times 73$ × $58 \mathrm{cms}$. Por su parte, los zócalos son similares, de $28 \times 73$ x $58 \mathrm{cms}$. y divididos en tres cuerpos, los dos primeros rectos de 12 y $4 \mathrm{cms}$ respectivamente, mientras que el tercero en forma de una gola inversa, tiene $12 \mathrm{cms}$. de altura. Todos ellos están acabados en su parte inferior por medio de un labio sin trabajar, para facilitar el engarce de las losas.

El alto grado de erosión de los textos, incrementado, además, por una posible damnatio de lín. 1, impide su lectura a simple vista. Por esta razón, ha sido necesario recurrir al uso de luz rasante desde diferentes ángulos para obtener la lectio ${ }^{7}$. Además, su lectura se ha realizado partiendo de la hipótesis, confirmada con posterioridad, de la existencia de un mismo dedicante para los cuatro pedestales, como así parecía intuirse de los pocos trazos apreciables a simple vista y de la homogeneidad del conjunto ${ }^{8}$. Esta circunstancia permitía plantear la posibilidad de que, cuando menos, la última línea del texto fuera similar en todos ellos, con la presencia del mismo promotor, lo cual posibilitaba reconstruir algunos caracteres irremediablemente perdidos.

Para finalizar esta breve presentación de los monumentos, conviene advertir que su exposición no se realizará siguiendo un orden topográfico, de izquierda a derecha, como sería lo esperable. Por el contrario, en aras de una mejor inteligibilidad del conjunto por parte del lector, el orden seguido se realiza en función del grado de conservación del texto. En cualquier caso, junto al número de ordenación se ha señalado, entre paréntesis, el número de orden topográfico.

1. (3). Base de arenisca local con el campo epigráfico rebajado. Muestra trazas de que se ha tratado de borrar la lín. 1. En el zócalo se aprecia un rebaje de $22 \mathrm{x}$ 27 cms. para sujetar una viga. Medidas: 123 x 73 x 58 cms. Campo epigráfico: 22 x 45 cms. Letras: 5. Capital cuadrada, con ciertos rasgos de libraria. Interpunción: punto (figs. 13 y 14).

$$
\begin{aligned}
& \text { A[-]O+[-]NIS } \\
& \text { POM PVLLA } \\
& \text { [-ca. 2-]S F PAVLLA H.E.T }
\end{aligned}
$$

\footnotetext{
6 Sobre el conjunto de zócalos conservados en el foro y la posibilidad de que se traten de pedestales tripartitos uid. JORDÁN LORENZO, Ángel A., «Inscripciones, monumentos anepígrafos, dudosos, sellos y grafitos procedentes del municipium ignotum de Los Bañales de Uncastillo", en: ANDREU PINTADO, Javier (ed.), La ciudad romana de Los Bañales (Uncastillo, Zaragoza): entre la historia, la arqueología y la historiografía, Caesaraugusta, 82 (2011), pp. 289-336, esp. 302-305.

7 Labor que se realizó durante sucesivas noches de los meses de Julio y Agosto de 2011 y Julio de 2012. En la noche del 27 de Julio acompañado por los Dres. F. Beltrán Lloris y J. Andreu Pintado y en la del 3 de Agosto por los Dres. J. Gómez-Pantoja y J. Andreu Pintado.

8 La disposición de conjuntos epigráficos financiados por un único donante es bastante conocida en la Península Ibérica, como pueden atestiguar, entre otros, el conjunto de Cocceia Severa en Capera (cf. GONZÁLEZ-CONDE PUENTE, Maria Pilar, "Cocceia Severa y los Coccei Hispanos», Hispania Antiqua, 24, (2000), pp. 165-173) o el de L. Aemilius Seranus en Andelo (CIL II 2966, CIL II 2967 y HEp 3, 263).
} 
En lín. 1 la crux corresponde a un trazo vertical, $I$ ó $L$. Esto permite plantear la posibilidad del desarrollo del teónimo como A[p]ol[i]ni. En relación con ello, el culto a Apolo encuentra su testimonio más cercano en la placa de bronce procedente de $A n d e{ }^{9}{ }^{9}$, y su difusión en la Península tiene lugar sobre todo en el siglo II d.C., acorde con el auge de los dioses salutíferos ${ }^{10}$. En lín. 3 nexo $A V$.

En lín. 2-3 se desarrolla el nombre de la evergeta, Pom(-) Pulla[-ca. 2-]s $f$. Paulla. El nomen Pom(--) puede desarrollarse en la forma Pom(ponia) o Pom(peia). Ambos casos aparecen atestiguados en la epigrafía peninsular. En el caso de Pomponius, -a, el texto más cercano aparece en Barbarín (Navarra) ${ }^{11}$, siendo el único caso constatado en el conuentus Caesaraugustanus. Por su parte, el nomen Pompeius, -a se encuentra disperso por todo el conuentus, siendo el testimonio más cercano el procedente de Arre ${ }^{12}$. En relación con ello, dada la amplia difusión del nomen Pompeius, -a en el conuentus, es posible plantear el desarrollo del nomen de Paulla como Pom(peia), con preferencia al de Pom(ponia).

El cognomen Pullas constituye un hapax en la Península Ibérica, aunque en Igaedis y Segobriga aparece registrada la forma Pullus y en Conimbriga Pulla ${ }^{13}$, mientras que sí que aparece en Tebessa ${ }^{14}$. Su origen es latino, y posiblemente se trate de una derivación de Pullus, cognomen alusivo a la «juventud» ${ }^{15}$.

A tenor de lo conservado y con las debidas reservas dado el pésimo estado de conservación del texto, quizá sea posible reconstruir la inscripción en la siguiente forma: A[p]ol[i]ni (?) s(acrum) / Pom(peia ?) Pulla/[ti]s f(ilia) Paulla h(eredes) $\cdot e(x)$ $\cdot t$ (estamento).

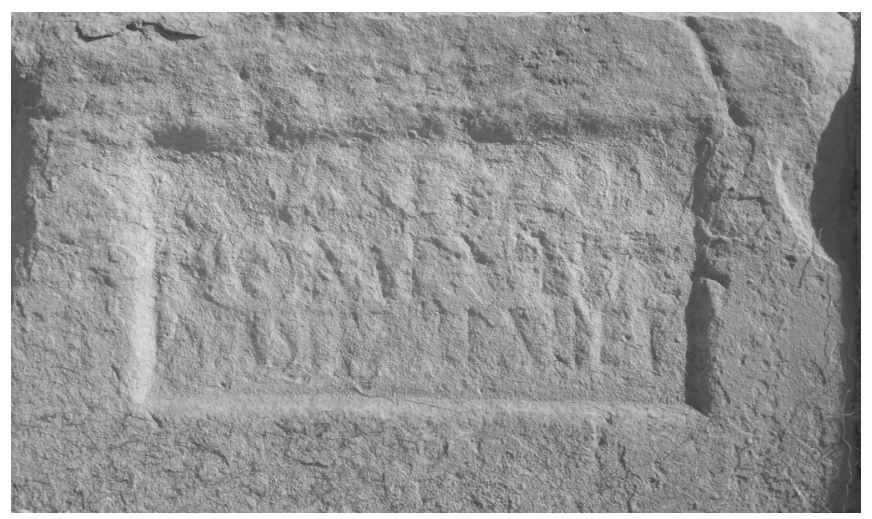

Fig. 9: Detalle campo epigráfico

${ }^{9}$ HEp 1, 491.

10 MANGAS, Julio, «El culto de Apolo en Hispania: testimonios epigráficos», en: Mélanges P. Léveque, 6, (1991), pp. 171-192, p. 190.

${ }^{11}$ IRMN 19.

12 CIL II 2958.

${ }^{13} \mathrm{CIL}$ II 286, 442 y HEp 9, 306, cf. VARIOS, Atlas antroponímico de la Lusitania romana, MéridaBurdeos, Ausonius, 2003, p. 274

${ }_{14}$ CIL VIII, 10624.

15 KAJANTO, liro, The latin cognomina, Helsinki, Helsingfors, 1965, p. 78. 


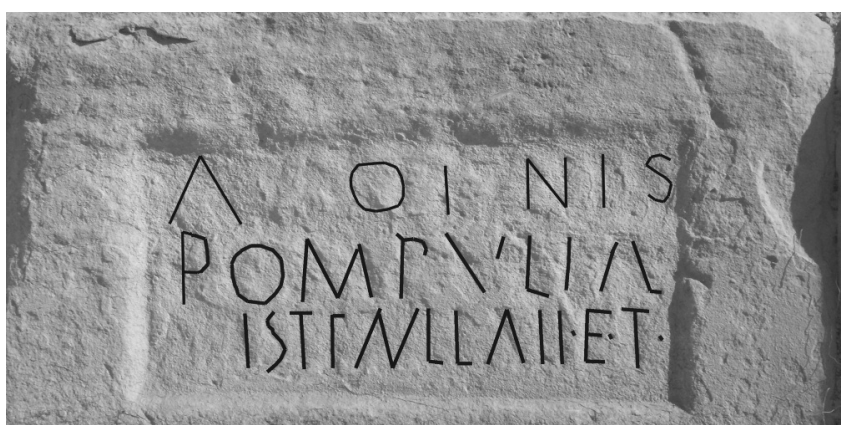

Fig. 10. Reconstrucción del texto

2. (2). Base de arenisca local con el campo epigráfico rebajado. Muestra una gran cruz en su lado izquierdo y trazas de que se ha tratado de borrar la lín. 1. Medidas: 123 x 73 x 58 cms. Campo epigráfico: 22 x 46 cms. Letras: 5 . Capital cuadrada, con ciertos rasgos de libraria. Interpunción: punto (figs. 11 y 12).

+ (uacat) IM+[-ca. 2-]S

POM PVLA+

TIS F PAVLLA $\cdot H \cdot E \cdot T$

En lín. 1 la primera crux corresponde a una $M$ ó $N$. Por desgracia, el estado del texto impide poder decantarnos por una u otra, pues no hay forma de dilucidar si el último trazo vertical, que formaría la $M$, se realizó conscientemente o si, por el contrario, se trata de una marca accidental. La segunda crux corresponde a un trazo vertical del que sale otro horizontal a media altura, posiblemente una $P$ ó $F$. En lín. 2 la crux corresponde al pie inferior de una letra. Se aprecia nexo PVL, conservándose la parte interior del trazo izquierdo de la $V$.

Por desgracia, el estado de conservación de lín. 1 no facilita la realización una lectura con fiabilidad. En nuestra opinión, los caracteres conservados quizá permitan reconstruir el teónimo [N]IMP[HI]S, cuyas atestiguaciones más cercanas se encuentran en Yesa y Barbarín (Navarra) ${ }^{16}$, con un epíteto abreviado delante que empezaría con la letra $M$, como la opción más viable ${ }^{17}$. La grafía del teónimo, con I latina en lugar de $Y$, aparece atestiguada también en Baños de Montemayor (Cáceres), León o Alongos (Orense), entre otros lugares ${ }^{18}$. Una alternativa a esta

16 HEp 11, 339 y AE 2002, 798.

17 En general, cuando aparece un epíteto caracterizando a las Ninfas, éste suele hacer referencia a un topónimo, como sucede con las Nymphis Caparensium (i. e. CPIL 76, 77) o las Nymphis Aur(iensium) (CIL II 2474, si bien se ha leído también como [N]ymhis (M(arcus)) Aur(elius), cf. DIEZ DE VELASCO, Fernando, Termalismo y religión. La sacralización del agua termal en la Península Ibérica y el norte de Africa en el mundo antiguo, Madrid, 1998, no 14/36), mientras que otras opciones mayoritarias pasan por aplicar la expresión sacrum (EE IX, 130; ERpLe 54), aludiendo a la dedicación del pedestal. En relación con el epíteto que pudo aplicarse en este caso, conviene, a modo de exempla señalar la existencia de unas Nym(phis) Mari(nis) en Canedo (Orense) (HEp 10, 388).

18 Baños de Montemayor: CPIL 73 (Nimphis Capa[r](ensium)); León: ErpLe 54 (Nimp\{p\}his sacr(um)); Alongos (Orense): NCIL II 5625 = IRG IV, 77 (Nimphis). 
posibilidad pasaría por considerar una deidad que se amoldara a la secuencia $M[-$ -JIMP[-ca. 2-]S, considerando que el espacio entre la $M$ y la I estuvo grabado, aunque en la actualidad se haya perdido, si bien no se ha encontrado, por el momento, algún teónimo que se ajuste a estos requisitos.

Con respecto a lín. 2, la existencia de un inicio POM $P$ similar al de la inscripción anterior, así como la semejanza de la lín. 3 en ambos textos, permite plantear con cierta verosimilitud que el espacio restante de este renglón estaría ocupado por el inicio del patronímico. Los trazos conservados, con un inusual nexo PVL, muestra como éste adopta la peculiar grafía Pul(I)astis, quizá por error del lapicida o por desconocimiento de su forma declinada, si bien no es posible precisar si adoptaría también esta forma en el resto de los textos.

De esta forma, con las reservas debidas al estado de conservación del texto, se propone la siguiente reconstrucción: $M(-)$ (?) [N]imp[hi]s (?) / Pom(peia ?) Pul(I)as/tis f(ilia) Paulla $\cdot h($ eredes $) \cdot e(x) \cdot t$ (estamento).

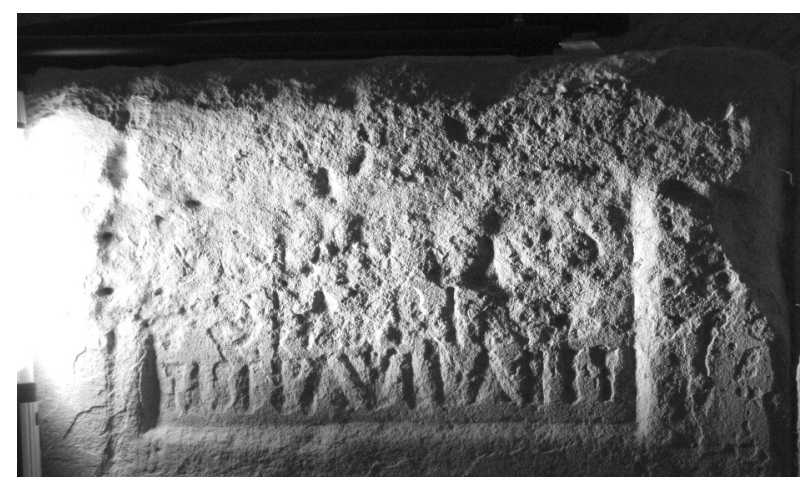

Fig. 11: Detalle campo epigráfico de la basis 2

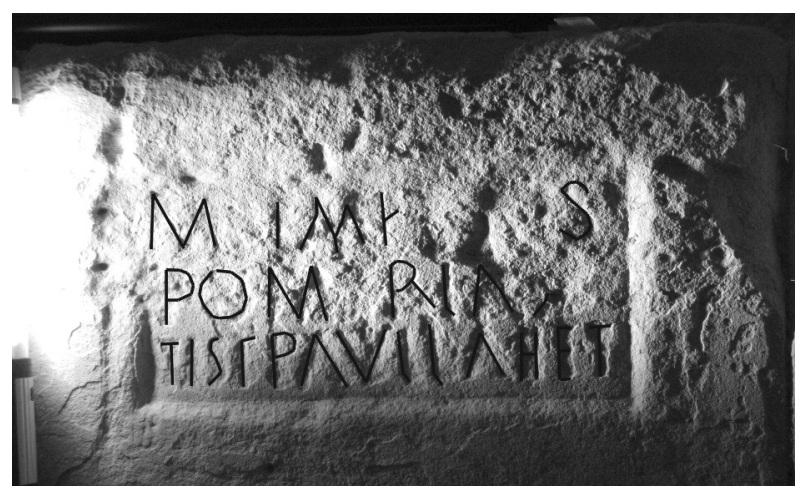

Fig. 12: Reconstrucción del texto de la basis 2 
3. (4). Base de arenisca local con el campo epigráfico rebajado. Muestra una pequeña fractura en su ángulo superior derecho, como consecuencia de haberse intentado levantar, aunque no afecta al texto. En su ángulo inferior izquierdo la moldura ha sido rebajada para hacer una cuna de $11 \times 24 \mathrm{cms}$., que continuaría en el siguiente pedestal. Medidas: $123 \times 73$ x $58 \mathrm{cms}$. Campo epigráfico: $20 \times 44 \mathrm{cms}$. Letras: 5. Capital cuadrada, con ciertos rasgos de libraria (figs. 15 y 16).

\section{MA[-]RII-]VS \\ TRIS POM \\ [-] + PAVLLA HET}

En lín. 1, es posible reconstruir el teónimo como Ma[t]ri[b]us, que hace referencia a las Matres, deidades indígenas, de origen celta y carácter salutífero, especialmente populares en las Galias y en ambas Germanias ${ }^{19}$. En lín. 2, la secuencia siguiente al teónimo, TRIS, quizá haga referencia al carácter triple de las Matres, planteándose como una mala grafía en lugar de tribus ${ }^{20}$. Otra opción pasa por considerar TRIS como el inicio de un topónimo, tal vez de origen de las Matres, aunque se desconoce cual pudo ser. En lín. 3, nexo AV y HE. La crux corresponde a un trazo vertical, posiblemente, por su disposición en el texto y a tenor de los otros conservados, una $F$. En el espacio existente al inicio de la línea, perdido como consecuencia de un golpe que propició la caída de una lasca, debió de ir grabado el patronímico Pullatis, como se aprecia en los otros textos, si bien tuvo que ser grabado con varios nexos para poder adecuarlo al espacio disponible.

En conclusión, a tenor de lo conservado, es posible reconstruir la inscripción en la siguiente forma: Ma[t]ri[b]us / Tris(—- ?) Pom(peia) / [Pullatis (?)] f(ilia) Paulla $h$ (eredes) e(x) t(estamento).

19 Sobre el culto a las Matres uid. RÜGER, Christoph B., «Beobachtungen zu den epigraphischen Belegen der Muttergottheiten in den lateinischen Provinzen des Imperium romanum», en: BAUCHHENSS, Gerhard y NEUMANN, Günter, Matronen und verwandte Gottheiten, Bonn, pp. 1-30. Para el caso hispano uid. GÓMEZ-PANTOJA, Joaquín, «Las madres de Clunia», VILLAR, Francisco y BELTRÁN LLORÍS, Francisco (eds.), Pueblos, lenguas y escrituras en la Hispania prerromana. Actas del VII Coloquio sobre lenguas y culturas paleohispánicas (Zaragoza, 12 a 15 de Marzo de 1997), Salamanca, Universidad de Salamanca, 1999, pp. 421-432 y OLIVARES PEDREÑO, Juan Carlos, Los dioses de la Hispania céltica, Madrid, Real Academia de la Historia - Universidad de Alicante, 2002, pp. 254-255.

20 En este sentido, se conoce en Condercum (Britannia) una consagración, realizada por T(-) Agrippa, praefectus del ala prima Hispanorum Asturum a las Matr(ibus) Tribus Campestrib(us), cf. CIL VII, $510=\mathrm{RIB} I, 1334$. 


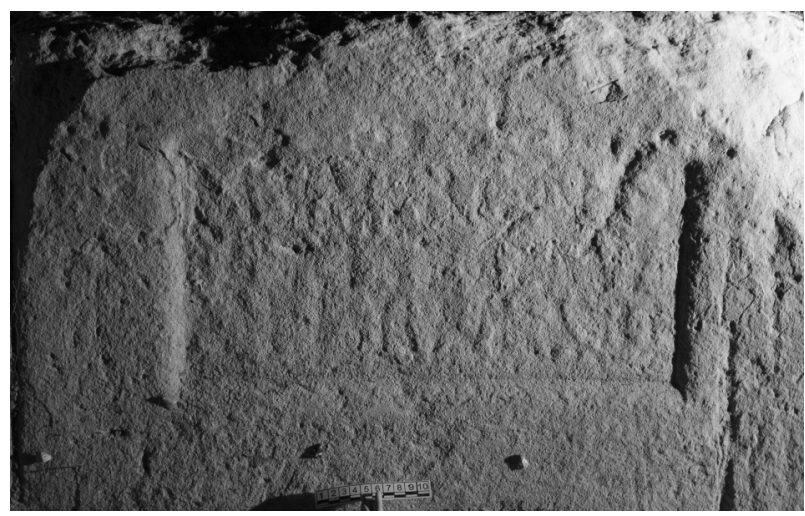

Fig. 13: Detalle campo epigráfico

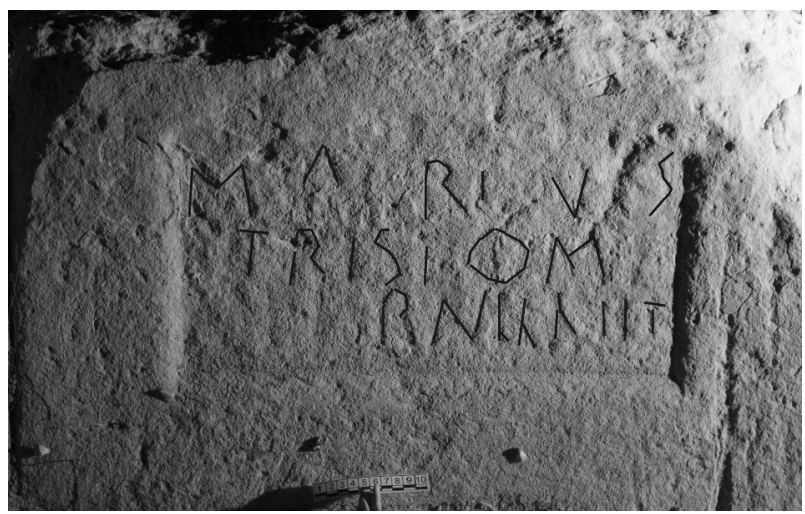

Fig. 14: Reconstrucción del texto

4. (1). Base de arenisca local cuyo bloque central muestra una fractura en su ángulo superior izquierdo, como consecuencia de un intento de expolio. Esto ha provocado la pérdida de parte del texto. Además, presenta en la parte derecha del zócalo una cuna de poste de $9 \times 10 \mathrm{cms}$, que ha reventado parte de las molduras. Medidas: 123 x 73 x 58 cms. Campo epigráfico: 22 x (43) cms., rectangular y rebajado. Letras: 5. Capital cuadrada (fig. 9 y 10).

$$
\begin{aligned}
& {[-- \text { - }]+[-] O V I} \\
& {[--] A V L L A+} \\
& H+T
\end{aligned}
$$

En lín. 1 la crux corresponde a un pequeño trazo diagonal, posiblemente de un pie, quizá de una $M$. Entre este trazo y la primera letra visible, la $O$, existe un espacio en el que, probablemente, debió haber trazado un caracter. Por esta razón, es posible desarrollar en lín. 1 el teónimo [I]ovi, precedido por una fórmula de consagración, quizá [Sacru]m. Su empleo en las consagraciones a Júpiter está so- 
bradamente atestiguado en la Península Ibérica, pudiéndose encontrar el paralelo más cercano en Ujué, en donde tres Coelii, Tesphoros, Festa y Telesinus levantaron un altar a esta deidad, acompañado por un segundo a Lacubegr21. Del mismo modo, el testimonio de culto a Júpiter no debe sorprender en un ambiente plenamente urbano, puesto que su culto aparece reglado en las leyes municipales ${ }^{22}$.

En lín. 2, es posible reconstruir el cognomen [P]aulla, si bien conviene reconocer que se aprecia un trazo vertical al final de la línea, quizá identificable con una raspadura. Otra opción, menos probable, es considerarlo como una I, pues no se aprecian restos que permitan pensar en una $E, R$ u otras similares. En este caso, se debería reconstruir el cognomen [P]aullai (sic) o, más probablemente, [P]aullae, si se admite una equivalencia $I=E$. En este sentido, la estilización de los trazos por parte de los lapicidas que actuaron en las Cinco Villas es conocida, como muestra la cupa de Caecilia Themi $f$. en donde las $T$ e I son similares ${ }^{23}$ y, a la vista del escaso sentido que tiene el cognomen [P]aullai, parecería más plausible pensar que se está ante un testimonio más de este mismo fenómeno. De aceptarse esta propuesta, se obtendría el cognomen Paulla declinado, de difícil explicación en este contexto, razón por la cual se opta por identificar, con toda la cautela debida al estado de la pieza, este trazo como una raspadura.

Paulla, como se ha comentado, es Pom(peia ?) Pullatis $f$. Paulla, donante de la construcción de la exedra, cuyo nombre aparece en el resto de textos. Por esta razón, no cabe descartar que el resto del nombre de la evergeta figurara en la parte perdida de lín. 2. Sin embargo, la diferente distribución del contenido del texto de esta inscripción con respecto a las restantes, obliga a tomar esta interpretación con precaución.

En lín. 3, la crux corresponde a un trazo vertical. A diferencia del resto de textos, se trata de una secuencia de tres letras, posiblemente identificables con la fórmula $h$ (eredes) $e(x) t($ estamento), si bien en este caso la $E$ se muestra completamente abrasada.

De esta forma, con las reservas debidas al pésimo estado de conservación, proponemos una reconstrucción del texto de la siguiente manera: [Sacru]m (?) [I]ovi / [-] [P]aulla (?) / h(eredes) e(x) (?) t(estamento).

21 IRMN 33 y 34.

22 A modo de curiosidad, cabe señalar que otra consagración a Júpiter se encontró en la vecina localidad de Asín, cf. ANDREU, Javier y JORDÁN, Ángel A., «Epigrafía, organización del territorio y poblamiento en territorio de vascones: Los Bañales (Uncastillo, Zaragoza)", ETF, Serie I, Prehistoria y Arqueología, 16-17, (2003-04), pp. 419-461, № 17, cuyo promotor debe leerse como Vi(bius) Turinnu[s], cf. HEp 5, 913 = AE 1997, 932.

${ }_{23}$ JORDÁN, Ángel A., ANDREU, Javier y BIENES, Juan José, «Epigrafía romana de Sofuentes», Epigraphica, 72, (2010), pp. 191-246, no 7. 


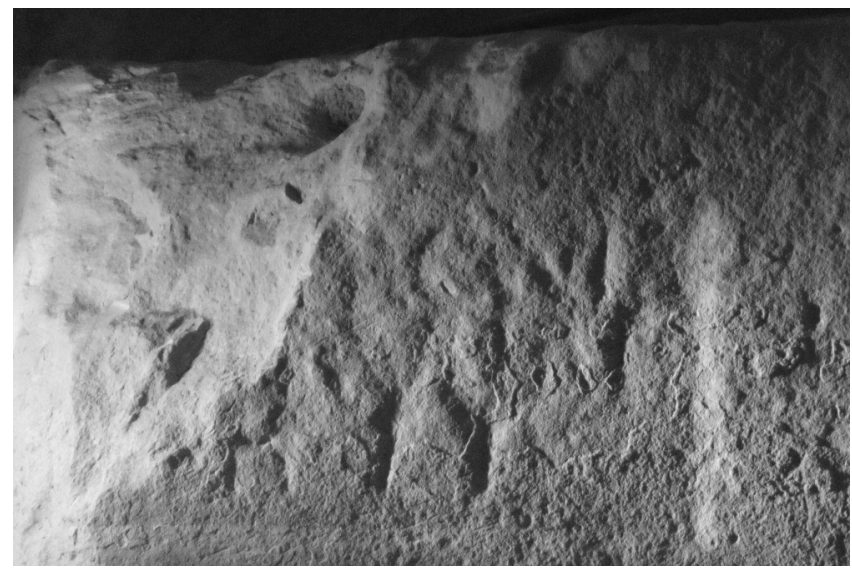

Fig. 15: Detalle campo epigráfico

De esta forma, para concluir, las inscripciones conservadas muestran la realización de un espacio de carácter sacro dedicado, al menos, a cuatro deidades. Por desgracia, su identificación no es segura. Con mayor claridad parecen reconocerse los teónimos de Júpiter y las Matres, mientras que se muestran más dudosas las identificaciones de Apolo y las Ninfas. En cualquier caso, muestran un marcado carácter salutífero y, además, en el caso de las Matres y las Ninfas, si es correcta la lectura de la lín. 1 de este texto, esta orientación se muestra muy vinculada con el agua. Por esta razón, resulta plausible interpretar este conjunto como la consecuencia de un voto que la evergeta, Pom(peia ?) Pullastis $f$. Paulla realizó en vida durante el transcurso de una enfermedad, para cuya curación acudió a un balneario o recinto termal emplazado en alguna zona celta, bien sea en Galia, Germania o el área celta de la Península Ibérica, pero que no pudo resolver en vida. La reiterada aparición de la fórmula $H E T$, muestra que su solución tuvo lugar tras su fallecimiento, por medio del cumplimiento de una manda que dejó establecida en su testamento.

Por otra parte, a través de la paleografía se aprecia que intervinieron, al menos, dos personas en la talla de las letras. Una primera las grabó empleando caracteres capitales cuadrados, dando cierta anchura al ductus, que se labró a doble bisel. El segundo lapicida realizó unas letras más estilizadas, casi librarias, con un ductus más sencillo y estrecho que, en ocasiones, da la sensación de ser semicircular, si bien el estado de conservación del texto impide confirmarlo con seguridad. Por el tipo de letra se puede datar este conjunto entre finales del siglo I d.C. y primera mitad del siglo II d. C.

Sobre el hecho en sí de esta actuación, conviene resaltar que la realización de donaciones o actos de evergetismo por parte de mujeres en la Península Ibérica supone una cuarta parte del volumen de testimonios conocidos de donaciones, si 
bien en la provincia Hispania Citerior representan tan sólo el 18\%24. Este fenómeno tendió a desarrollarse desde mediados del siglo I d. C., adquiriendo su cenit en el siglo II d. C. ${ }^{25}$, con los objetivos de obtener, mantener o acrecentar el prestigio y la posición social, tanto personal como familiar, perpetuando la memoria de la gens en el conjunto de la sociedad.

Centrando la atención en monumentos similares a la extraordinaria donación de Pom(peia ?) Pullatis $f$. Paulla, conviene subrayar que la consagración de monumentos de carácter cultual ex testamento no es un fenómeno demasiado frecuente en la Península Ibérica, pues hasta el momento se conocen 34 testimonios $^{26}$, lo cual apenas supone un $1 \%$ del conjunto de la epigrafía cultual datada durante el Principado en la Península Ibérica. Dentro de este conjunto de textos destacan sendas legaciones realizadas por L. Minicius Apronianus, que afectó a varias localidades, y por L. Porcius Victor, en Cartima.

L. Minicius Apronianus, magistrado de Tarraco, dejó establecido tras su muerte la realización de, al menos, tres estatuas de diferente carácter ${ }^{27}$. La primera de ellas fue una consagración al Genio de la Coloniae Iuliae Urbis Triumphalis Tarraconensium consistente de una estatua de plata de 15 libras de peso. La segunda, encontrada en las termas de Aquae Calidae, consistió en una estatua a Apolo. Por último, las disposiciones de su testamento incluyeron, al menos, una estatua de él mismo, que debió de emplazarse en su uilla.

Por su parte, del municipio Flavio de Cartima proceden dos textos, uno de ellos perdido, dispuestos en el testamento de L. Porcius Victor ${ }^{28}$. El primero de ellos se trata de una consagración a Marte Augusto, en donde se especifica que los herederos no dedujeron el cinco por ciento de la $X X$ hereditatium y realizaron un epulum. En el segundo, correspondiente a un pedestal de mármol, L. Porcius Victor levantó una estatua a Venus Augusta, tanto en su nombre como en nombre de su

${ }^{24}$ MELCHOR GIL, Enrique, «Mujeres y evergetismo en la Hispania romana», en RODRíGUEZ NEILA, Juan Francisco (cur.), Hispania y la epigrafía romana, cuatro perspectivas, Faenza, Epigrafia e Antichità 26, 2009, pp. 133-178, p. 136.

25 MELCHOR GIL, Enrique, «Mujeres y evergetismo...», p. 175.

26 Baetica: CIL II $1424=\mathrm{CIL} \mathrm{I} / 2 / 5,853$ y CIL II $1425=\mathrm{CIL} \mathrm{II} / 5,854$ de Cortijo del Tajo, CIL II 1473 $=\mathrm{CIL} \mathrm{I}{ }^{2} / 5.1164$, CIL II $1474=\mathrm{CIL} \mathrm{I} / 5.1165$ y CIL II²/5. 1166 de Astigi, CIL II $1637=\mathrm{CIL} \mathrm{II2/5.} 273$ de Ipolcobulcula, CIL II $1663=\mathrm{CIL} \mathrm{II} / 5.69$ de Tucci, CIL II $2006=\mathrm{CIL} \mathrm{II} / 5.838$ y CIL II $2007=\mathrm{CIL} \mathrm{II²} / 5.839$ de Nescania, CIL II $2034=$ CIL II²/5. 742 de Anticaria, CIL II $1036=$ CIL II²/7, 975 de Regina, CIL II 2244 $=\mathrm{CIL} \mathrm{II} / 7,229$ de Corduba, CIL II $5513=\mathrm{CIL} \mathrm{I} / 2 / 7.96$ de Obulco, CIL II2/7. 138 de Epora, CIL II 1949, 1951 y 1952 de Cartima, CIL II 1062 = CILA II, 221 de Arua, CIL II 1166 = CILA II, 4 de Hispalis, CIL II 2326 = CILA II, 165 de Celti, CILA II, 1057 de Munigua, CILA II, 233 de Canania, CILA II, 1012 de Laelia Iulia. Lusitania: EE VIII, 14 de Fundao (Castelo Branco), CIL II 21 = IRCP 144 de Mirobriga, HEp 15, 488 de Conimbriga. Hispania Citerior. CIL II 3265 = CILA III, 80 de Castulo, CIL II 3411 = DECar 40 y CIL II 3424 = DECar 60 de Carthago Noua, CIL II 2819 = ERSo 20 de Uxama Argaela, CIL II 5816 de Veleia, CIL II $3819=$ CIL II2/14. 291 de Saguntum, CIL II $4071=$ RIT 23 de Tarraco, CIL II $4488=$ IRC I, 34 de Aquae Calidae. En general, sobre las donaciones ex testamento uid. MELCHOR GIL, Enrique, «Evergetismo testamentario en la Hispania romana: legados y fundaciones», MHA, 15-16, (1994-95), pp. 215228.

27 CIL II $4274=6072$ = RIT 918, cf. GOROSTIDI PI, Diana, Ager Tarraconensis 3. Les inscripcions romanes, Tarragona, ICAC, 2010, no 8; CIL II 4488 = IRC I, 34 y CIL II 4071 = RIT 23.

28 CIL II 1949 y CIL II 1951. 
esposa, Scribonia Marciana, completando la donación con la noticia de la realización del epulum y de ausencia de la deducción de la XX hereditatium.

Para finalizar, dentro de este conjunto de donaciones de carácter cultual realizadas ex testamento, sólo en el caso de nueve de ellas $(26,4 \%)$ fueron legaciones dispuestas por una mujer ${ }^{29}$. Sin duda, la escasa cantidad de inscripciones conservadas muestran que son acciones claramente excepcionales, especialmente en Hispania Citerior, de donde sólo proceden dos textos: un pedestal recubierto por placas de bronce realizado por Pompeia L. f. Moderata en honor de Mercurio Augusto y una estatua de cien libras de plata consagrada a la Pietas realizada por Cornelia C. f. Marullina en memoria de su hijo L. Cornelius Marullus en Castulo ${ }^{30}$.

Un segundo aspecto a resaltar, además de la singularidad de la donación en sí, es su emplazamiento en el foro de la ciudad romana de Los Bañales. El hallazgo de los textos in situ permite apreciar la ocupación del espacio público por parte de un programa iconográfico/epigráfico claramente privado. Esta misma circunstancia se aprecia en el segundo programa epigráfico encontrado in situ en el foro de Los Bañales, financiado por M. Fabius Nouus y Porcia Fauentina, actualmente en proceso de estudio. Sin duda, en ambos casos se puede comprobar, de primera mano, el recurso a la construcción de un espacio, en esta ocasión de carácter sacro, con una finalidad claramente publicitaria. En este sentido, el recurso a la construcción evergética permitía al interesado tener un espacio escenográfico relativamente amplio y con pocas limitaciones legislativas, lo cual le permitía mostrar su preeminencia a la sociedad, en un ambiente público, aspecto sobre el que ya se incidió en otro lugar ${ }^{31}$.

Estos datos, sin duda, permiten subrayar el carácter inusual del conjunto que ha ocupado estas páginas, financiado por Pom(peia ?) Pullatis f. Paulla. Por desgracia, su deficiente estado de conservación impide apreciar con total claridad el contenido de los pedestales a simple vista, pero no cabe duda de que su hallazgo in situ suponen una extraordinaria aportación, tanto al conocimiento de la sociedad de la desconocida ciudad de Los Bañales de Uncastillo, como al de la disposición epigráfica urbana en general.

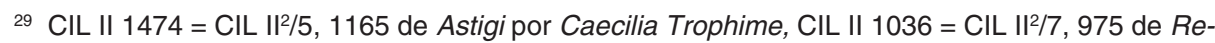
gina por Terentia Puella, CIL II 1951 por L. Porcius Victor y Scribonia Marciana y CIL II 1952 por Vibia L. f. Rusticana, ambas de Cartima, CIL II 2326 = CILA II, 165 de Celti por M. Annius Celtitanus y Aemilia Artemisia de Celti, CILA II, 1057 de Munigua por Fabia Ursina, CILA II, 233 de Canania por Sempronia Galla, CIL II 3265 = CILA III, 80 de Castulo por Cornelia C. f. Marullina y CIL II $2819=$ ERSo 20 de Uxama Argaela por Pompeia L. f. Moderata.

30 CILA III, 80 y CIL II 2819 = ERSo 20.

${ }_{31}$ JORDÁN, Ángel A., «Algunos condicionantes estructurales a la disposición epigráfica en la ciudad romana hispana», en: ANDREU PINTADO, Javier, CABRERO PIQUERO, Javier, RODÀ DE LLANZA, Isabel (eds.), Hispania: las provincias hispanas en el mundo romano, Tarragona, ICAC, 2009, pp. 125138, esp. 133-137. 
